\title{
The Influence on Airflow Characteristics In Upper Airway In Sleep Apnea Hypopnea By The Disturbance of Pharyngeal Muscle Group Based on High Simulation of The Wall Flow Field
}

\section{Xinyan HUANG}

Jingan District ZhaBei Central Hospital,Shanghai https://orcid.org/0000-0001-9173-4963

Fan PENG ( $\sim$ pf6666@126.com )

Jingan District ZhaBei Central Hospital,Shanghai https://orcid.org/0000-0002-8466-786X

\section{Liren SUN}

Jing'an District Zhabei Centre Hospital of Shanghai

Jie BI

Jing'an District Zhabei Centre Hospital of Shanghai

Jue CHEN

Jing'an District Zhabei Centre Hospital of Shanghai

Ting ZHANG

Jing'an District Zhabei Centre Hospital of Shanghai

Huanhuan CHEN

Jing'an District Zhabei Centre Hospital of Shanghai

\section{Liangzhen WU}

Jing'an District Zhabei Centre Hospital of Shanghai

\section{Research article}

Keywords: The disturbance of pharyngeal muscle group, Obstructive sleep apnea hypopnea syndrome (OSAHS), injection sclerotherapy, submucosal injection edema, Computational fluid dynamics, Threedimensional reconstruction

Posted Date: February 23rd, 2021

DOl: https://doi.org/10.21203/rs.3.rs-247839/v1

License: (c) (1) This work is licensed under a Creative Commons Attribution 4.0 International License. Read Full License 


\section{Abstract}

Background: To observe the influence on morphology and internal airflow characteristics in upper airway in sleep apnea hypopnea by the disturbance of pharyngeal muscle group based on high simulation of the wall flow field.

Methods: One goat for experimental purpose bought by our hospital in December 2018 was included as the research object. This experimental goat received injection of hardener and submucosal injection edema into pharyngeal muscle group of upper respiratory tract. The goat received CT scan before and after injection. Computational fluid dynamics(CFD) model was built on the base of CT scans by 3Dslicer 4.5 and MeshLab and ANSYS ICEM CFD 14.0. The internal flow of upper respiratory tract was simulated by ANSYS-FLUENT 14.0 and the results were analyzed by ANSYS-CFD-POST 14.0.

Results: Soft palate and uvula were elongated and thickened and pharyngeal muscle group was disturbed after injection of hardener and submucosal injection edema into the goat. The area that changed the most of upper airway was located in the lower bound of pharyngopalatiae, and it reduced area from $0.3602 \mathrm{~cm} 2$ to $0.1699 \mathrm{~cm} 2$; the air velocity was elevated from $3.53009 \mathrm{~m} / \mathrm{s}$ to $7.24478 \mathrm{~m} / \mathrm{s}$, the negative pressure was elevated from $-28.6184 \mathrm{~Pa}$ to $-66.4510 \mathrm{~Pa}$, while the resistance of cavum pharyngis elevated from 3396.09Pas/L to 3813.65Pas/L.

Conclusion: Injection of hardener and submucosal injection edema into pharyngeal muscle group of the goat would result in the disturbance of pharyngeal muscle group, narrow the upper respiratory tract, elevate the negative pressure and resistance of the upper respiratory tract in this area, bring about the collapsibility of the upper airway, obstruct the patency of the airflow and form the pathological model of OSAHS at last.

\section{Background}

Obstructive sleep apnea hypopnea syndrome(OSAHS) is caused by obstruction of upper airway during sleep ${ }^{[1]}$. The incidence of this disease is gradually increasing in recent years, which is closely related to the improvement of people's living standards, the development of medical diagnostic technology and people's increasing attention to health. The study on the etiology of OSAHS has been extended to the entire upper respiratory tract, the coordination of pharyngeal muscle group, the influence of secretion environment and the role of respiratory airflow with shape resistance, all of which are being gradually incorporated into the etiologic research.

With the swift development of information technology, the combination of medicine and engineering has become the future direction, so computer simulation has become one of the important methods for theoretical and experimental research, and the relevant methods of human body test can be replaced by computer simulation in some fields completely ${ }^{[2]}$. Three-dimensional images of respiratory tract can be well applied in the evaluation of OSAHS surgical efficacy ${ }^{[3]}$. Computational Flow Dynamics(CFD) ${ }^{[4,5,6]}$ needs to be combined with clinical medicine and clinical statistics effectively to conduct scientific and 
reasonable sorting, analysis and calculation for first-line clinical data, so as to assist clinical research with more accurate data. Through the study of three-dimensional reconstruction and CFD before and after injection of hardener and submucosal injection edema into goats' pharyngeal muscle group of upper respiratory tract, this paper investigates the results of upper respiratory tract morphology, flow velocity, pressure and airway resistance caused by disturbance of pharyngeal muscle group, so as to qualitatively and quantitatively analyze the effect of the functional status of pharyngeal muscle group to OSAHS and explore a new treatment on the basis of pharyngeal muscle group adjustment.

\section{Materials And Methods}

2.1 Selection of research objects One male goat aged 2.7 years old with the weight of $30.42 \mathrm{~kg}$ (from Shanghai Jiagan Biotechnology Co., LTD.) purchased December 2018 by our hospital was selected. It has no a history of acute and chronic disease in upper respiratory tract, trauma and surgery in maxillofacial region and upper respiratory tract within the last 3 months.

2.2 Methods The goat was performed with injection of hardener and submucosal injection edema in the bilateral soft palates in lower bound of uvula in pharynx, pharyngeal muscle groups in the upper, middle and lower ends of left and right pharyngopalatiaes( $3 \mathrm{ml}$ of $23.4 \%$ sodium chloride solution each). 3D CT scan of the upper respiratory tract was performed 1 week before and after the injection.

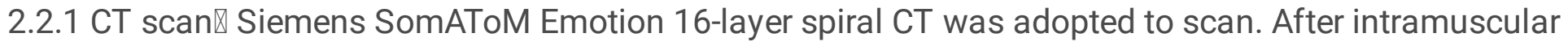
injection of Zoletil, under general anesthesia, goats were in supine position and breathed smoothly with head keep in midline by headstock. From $3 \mathrm{~cm}$ above the bottom of skull to supraglottic structure were scanned with $1.25 \mathrm{~mm}$ of scanning layer. Standard DICOM imaging data was collected by the software of Siemens CT machine.

2.2.2 Three-dimensional reconstruction of the upper respiratory tract $\ C T$ scanned data were used by 3Dslicer 4.5 to conduct three dimensional reconstruction. After treatment such as threshold value setting, image segmentation and region growth, three dimensional surface mathematical models were obtained, and the smooth outer surface was obtained with algorithm of MeshLab(figure 1). The model storage capacity was reduced and stored as STL format.

2.2.3 Meshing and independence analysis of three dimensional model The three dimensional model in STL format after smooth processing in the previous step was imported into the CFD pre-processing software ANSYS ICEM CFD 14.0 to conduct fluid mesh division, define upper airway inlet and outlet and build tetrahedral unstructured mesh model.

Before the next CFD calculation, the independence of mesh was firstly analyzed to ensure that the calculation data obtained were grid-independent, and then the next CFD calculation was carried out. The same flux flow at inlets could be set with different numbers of mesh such as 600,000, 1.2 million, 1.65 million and 2 million to calculate the upper respiratory tract. With quality and speed considered overall, the comparative results showed that the accuracy of numerical simulation was directly related to the 
quality of mesh division. The more the meshes, the more accurate the calculated results would be. However, in the comparison between 1.65 million and 2 million of meshes, the calculated results were basically the same except for the slight difference in local areas. Through the above analysis, it can be concluded that when the mesh density reaches more than 1.65 million, the calculation result is irrelevant to the number of meshes, and accurate calculation can be carried out in the next step. In this paper, the upper respiratory tract model with 1,751,940 of meshes and 303,981 of nodes was selected before and after injection.

2.2.4 Fluid mechanics parameters and boundary conditions With CFD method to calculate the internal air flow of upper respiratory tract, calculation domain was from the nostril to the bottom of the epiglottis. Software ANSYS- FLUENT 14.0 was adopted to conduct numerical simulation and SIMPLEC method was used to calculate the coupling of the velocity and pressure. On the basis of given pressure field, discrete Navier-Stokes momentum equation can be solved so as to obtain velocity field. Convergent solution can be calculated through repeated iteration. The whole upper respiratory tract is regarded as a rigid body with air as the fluid(normal temperature and incompressible). The thermal effect between fluid and wall surface was removed. The air flowed into the nose at a constant speed and the direction was vertical to the axial plane of inlet. With other conditions unchanged and RNG $k-\varepsilon$ turbulent-flow model to stimulate, it can be analyzed that the fluid flow in the upper respiratory tract was turbulent flow of low Reynolds number.

Boundary conditions: the upper respiratory tract was considered as a cavity, and the anterior nostril was directly connected with the outside environment, and a standard atmospheric pressure was applied at the nostril, that is, $\mathrm{P}=101325 \mathrm{~Pa}$; No slip with zero velocity was set on the surrounding wall surface, and the lower edge of throat was defined as the flow boundary condition with $120.7 \mathrm{ml} / \mathrm{s}$ or $7.242 \mathrm{~L} / \mathrm{min}$ of flow. Air was adopted as medium with $\rho=1.225 \mathrm{~kg} / \mathrm{m}^{3}$ of density and $\mu=1.7894 \times 10^{-5} \mathrm{~kg} /(\mathrm{m} \cdot \mathrm{s})$ of dynamic coefficient of viscosity. The Kinetic energy $k$ and turbulent dissipation rate $\varepsilon$ were calculated by the following formula:

$k=3 / 2(v l)^{2} \varepsilon=\mathrm{C}_{\mu}{ }^{0.75} \mathrm{k}^{3 / 2} / 0.07 \mathrm{~L}$

where: $v$. average speed; $l$ : turbulence intensity $l=0.16 \operatorname{Re}^{\wedge}(-1 / 8) ; \operatorname{Re}$ : Reynolds number $R e=v d \rho / \mu$; $d$. caliber; $\rho$ : medium density; $\mu$. dynamic viscosity coefficient of medium; $\otimes \mathrm{C}_{\mu}{ }^{0.75}=0.07$; L: characteristic length

2.2.5 Selection of model section The pharyngeal cavity was divided into 4 segments from top to bottom ${ }^{[7]}$ : nasopharynx (from the top of nasopharyngeal to horizontal hard palate), palatopharynx (from the horizontal hard palate to the tip of soft palate), glossopharynx (from the tip of soft palate to the tip of epiglottis), laryngopharynx (from the tip of epiglottis to the bottom of epiglottis ). 5 sections were selected as shown in the figure(figure 2): Section 1 was the beginning and merging area of the two nasal cavity, forming the end of nasal cavity and the beginning of the nasopharynx (nasopharyngeal apex); Section 2 was the lower nasopharyngeal boundary (horizontal hard palate); Section 3 was the 
subpalatopharyngeal boundary (the tip of soft palate); Section 4 was the bottom boundary of glossopharyngeum (the tip of epiglottis); Section 5 was the lower boundary of epiglottis (the bottom of epiglottis).

2.2.6 Result analysis The changes of upper respiratory tract morphology and air flow characteristics of goats' pharyngeal muscle group before and 1 week after injection were compared.

\section{Results}

3.1 Morphological changes of upper respiratory tract

As shown in table 1 and table 2, the volume of each region and the area of each section of goat pharyngeal muscle group before and after injection were calculated. There was no large volume change of nasopharynx and throat after the injection of hardener and submucosal injection edema, but disturbance of pharyngeal muscle groups occurred after the injection. The main changes occurred at the glossopharyngeum(reduced by $37.28 \%$ ). The section 3 area(the lower bound of pharyngopalatiae) of the upper respiratory tract of the goat shank obviously by $52.83 \%$ after injection.

Table 1 Changes in airway volume of pharyngeal muscle group before and after injection of hardener and submucosal injection edema $\left(\mathrm{cm}^{3}\right)$

\begin{tabular}{lccc}
\hline \multicolumn{1}{c}{ Area } & Before & After & Gradient \\
\hline Nasopharynx & 2.2051 & 2.1747 & $1.38 \%$ \\
Pharyngopalatiae & 5.3001 & 5.1245 & $3.31 \%$ \\
Glossopharyngeum & 0.8085 & 0.5071 & $37.28 \%$ \\
Laryngopharynx & 1.5940 & 1.5735 & $1.28 \%$ \\
Total volume & 9.9077 & 9.3798 & $5.33 \%$ \\
\hline
\end{tabular}

Table 2 Change of area of each section $\left(\mathrm{cm}^{2}\right)$ of pharyngeal muscle group before and after injection of hardener and submucosal injection edema

\begin{tabular}{cllc}
\hline Section & Before & After & Gradient \\
\hline 1 & 2.2369 & 2.2251 & $0.53 \%$ \\
2 & 3.0180 & 2.9187 & $3.29 \%$ \\
3 & 0.3602 & 0.1699 & $52.83 \%$ \\
4 & 0.3283 & 0.2863 & $12.79 \%$ \\
5 & 1.6056 & 1.6017 & $0.24 \%$ \\
\hline
\end{tabular}


The change trend of flow velocity in the upper respiratory of the goat before and after injection was shown in figure 3: Before injection, the airflow velocity in their upper respiratory tract was relatively stable but changed dramatically after injection. As shown in table 3 , there was no significant change in the airway velocity in the nasopharynx after injection, while the flow velocity in pharyngopalatiae and glossopharyngeum increased significantly, especially the lower bound of pharyngopalatiae, with an increase of $105.23 \%$.

Table 3 Change of flow velocity $₫ \mathrm{~m} / \mathrm{s} \llbracket$ of each airway section before and after injection of hardener and submucosal injection edema into pharyngeal muscle group

\begin{tabular}{cllc}
\hline Section & Before & After & Gradient \\
\hline 1 & 0.557899 & 0.560961 & $0.55 \%$ \\
2 & 0.681545 & 0.694548 & $1.91 \%$ \\
3 & 3.53009 & 7.24478 & $105.23 \%$ \\
4 & 3.73472 & 4.26934 & $14.31 \%$ \\
5 & 2.00396 & 2.15943 & $7.76 \%$ \\
\hline
\end{tabular}

\subsection{Pressure change in upper respiratory tract}

Pressure change trend of upper respiratory tract before and after injection were shown in figure 4 and table 4: the pressure of the upper respiratory tract decreased gradually along the direction of gas flow before injection. The pressure change in the nasopharynx and epiglottic areas was not significant after injection, while the negative pressure at the pharyngopalatiae and glossopharyngeum was significantly increased, especially the lower bound of pharyngopalatiae, from -28.6184Pa before injection to $-66.4510 \mathrm{~Pa}$, with a $132.20 \%$ increase.(take pressure of nasal cavity's inlet as the reference point: here the pressure is $101325 \mathrm{~Pa}$ ).

Table 4 Pressure change of each airway section before and after injection of hardener and submucosal injection edema into pharyngeal muscle group $(\mathrm{Pa})$ 


\begin{tabular}{cccc} 
Section & Before & After & Negative pressure gradient \\
\hline 1 & $101322.3832(-2.6168)$ & $101322.4324(-2.5676)$ & $-1.88 \%$ \\
2 & $101322.1633(-2.8367)$ & $101322.1853(-2.8147)$ & $-0.78 \%$ \\
3 & $101296.3816(-28.6184)$ & $101258.5490(-66.4510)$ & $132.20 \%$ \\
4 & $101291.8960(-33.1040)$ & $101269.0747(-55.9253)$ & $68.94 \%$ \\
5 & $100912.4750(-412.5250)$ & $100862.1240(-462.8760)$ & $12.21 \%$ \\
\hline
\end{tabular}

\subsection{Resistance change in upper respiratory tract}

Airflow resistance refers to the pressure required to push the specific volume of airflow to the unit distance in unit time. The resistance formula: $R=\triangle P / Q, \triangle P$ is pressure drop; $Q$ is flow, $0.1207 \mathrm{~L} / \mathrm{s}$. The cavum resistance of pharyngeal muscle group increased significantly after injection, from 3396.09Pa.s/L before injection to $3813.65 \mathrm{~Pa} \cdot \mathrm{s} / \mathrm{L}$, and the airflow resistance of pharyngeal muscle group increased $12.30 \%$ after injection.

\section{Discussion}

4.1 Establishment of the high simulation of the wall flow field in the upper respiratory tract $\$

Under a condition of smooth breathing, Kelly et al. ${ }^{[8]}$ compared the obtained data recorded in continuous time and found that the air flow was stable. Hahn ${ }^{[9]}$ found through experiments that the nasal hair had no effect on the internal air flow in a 20 -fold magnification model of nasal cavity. With the analysis of heat and mass transfers and the verification of Prandtl and Grashof numbers, it can be further concluded that under normal breathing conditions, temperature and humidity have no significant influence on the internal air flow. It is reasonable to regard the upper respiratory tract model in this study as instantaneous rigid model and the airflow in it is stable according to previous studies without the influence of nose hair and the change of temperature and humidity, therefore, these simplifications are reasonable.

Three-dimensional reconstruction and numerical simulation analysis of the upper respiratory tract on the basis of CT and MRI imaging in recent years has become increasing prevalent for scholars home and abroad $^{[10-12]}$. Based on CT scan and three dimensional reconstruction of upper respiratory tract before and after injection into pharyngeal muscle group, this study reconstructed the simulative structure of goat's respiratory tract with computer. Whereafter commercial software CFD was conducted to systematically calculate and analyze the flow field parameters of the upper respiratory tract. From the viewpoint of fluid mechanics, discussion and analysis of characteristics of upper respiratory air flow was conducted and analyzed from the anatomical structure with the flow characteristics of the upper respiratory tract taken into consideration. In this way, the relationship between anatomical structure, aerodynamic characteristics and physiological function of the upper respiratory tract can be more 
completely linked. The upper respiratory tract model was not simplified geometrically in the modeling process to establish high simulation of the wall flow field which could reflect the real situation of it.

\subsection{Discussion on CFD and characteristics of upper airway flow field}

In the field of biomedical engineering, Computational Flow Dynamics (CFD) is a new area of OSAHS research. The wall flow field with high simulation of upper respiratory tract was established through the above experimental goats, and some indexes of CFD analysis could reflect the relationship between related upper respiratory tract airflow and structure, which makes up for the difference between animal tissue structure and human's. Compared with the current commonly used detection methods, it can be found that this method can provide more accurate upper airway fluid data, and CFD technology has the advantages of fast modeling, high accuracy, no wound and repeatability.

The reference values of CFD study on upper respiratory tract include the normal range of cross sectional area, volume, velocity, pressure and wall resistance of upper respiratory tract. The effective ventilation volume of the upper respiratory tract is directly related to the degree of airway patency, belonging to an important index ${ }^{[13,14]}$. The respiratory function of the upper respiratory tract will be significantly affected when the effective ventilation volume is reduced. For example, the effective ventilation volume will decelerate the airflow speed in the middle of respiration. However, the reduction of this volume ${ }^{[15]}$ will also have a certain impact on the oxygen saturation capacity of the lower airway, and in severe cases, will result in basic diseases as well. From morphology, airflow distribution, velocity and pressure characteristics, it can be concluded that the impact force of airflow is different in different parts of upper respiratory tract when breath

It is convenient to identify the characteristic value parameters of flow field using CFD to simulate the air flow within the upper respiratory tract so as to know air flow situation and analyze different pressure in upper respiratory tract from three-dimensional direction. The blocked plane and its serious degree can be speculated with statistical analysis of mathematical software, which conduces to learning relationship between the function of upper respiratory tract and anatomical structure. Therefore, it provides a brandnew method to study the pathogenesis of OSAHS and explore the OSAHS mechanism caused by the disturbance of pharyngeal muscle group. In this study, CFD simulation was carried out on the three dimensional models of upper respiratory tract before and after injection of hardener and submucosal injection edema in one goat's pharyngeal muscle groups to study the changes of airflow velocity, pressure and wall resistance in upper respiratory tract after injection of hardener caused disturbance of pharyngeal muscle groups, and the morphological changes of upper respiratory tract were analyzed.

\subsection{Changes of upper respiratory tract after disturbance of pharyngeal muscle group}

In this article, according to the test data, we established three dimensional model of goats' and high simulation of the wall flow field, analyzed the change of upper respiratory tract before and after injection of hardener and submucosal injection edema into pharyngeal muscle group, adopted CFD software to determine each parameter value of flow field of the upper respiratory tract under smooth breathing. To 
sum up, without interference factors, this paper systematically expounds the impact on upper respiratory tract, because of the change of anatomical structure, posed by the disturbance of pharyngeal muscle group.

The numerical models of upper respiratory tract of the goat's pharyngeal muscle group before and after injection of hardener and submucosal injection edema were analyzed in detail. A comparative study found that the structure of the upper respiratory tract changed after injection, and the corresponding hydromechanical features changed significantly. After injection, there was almost no change in nasal cavity and laryngopharynx, while the area of pharyngopalatiae and glossopharyngeum were significantly shrunk; The air flow of the upper respiratory tract was affected due to the reduction of volume, and the velocity of the this area became rapid, especially the lower bound of pharyngopalatiae with velocity increased from $3.53009 \mathrm{~m} / \mathrm{s}$ to $7.24478 \mathrm{~m} / \mathrm{s}$. It aggravated the impact force of airflow on the pharyngeal wall and the damage to the airway mucosa. At the same time, the impact force of airflow on the wall of the pharynx cavity caused high-frequency vibration to soft tissues and the occurrence of snoring, and the enhancement of this impact force rose the snoring accordingly. After injection of hardener and submucosal injection edema into pharyngeal muscle group, the negative pressure in the lower bound of pharyngopalatiae was significantly increased from -28.6184Pa before injection to $-66.4510 \mathrm{~Pa}$, with a $132.20 \%$ increase, which enhanced the airway compliance and would increase the possibility of airway collapse in this area, leading to OSAHS symptoms. After injection of hardener and submucosal injection edema into pharyngeal muscle group, pharyngeal cavity resistance increased significantly by $12.30 \%$, which would make air flow through the airway more tough and further increase the possibility of collapse.

\section{Conclusion}

Pharyngeal cavity is the portal of the upper respiratory tract and its stable structure is closely related to respiration. Disturbance of pharyngeal muscle group and constriction of upper respiratory tract occurred after injection of hardener and submucosal injection edema into pharyngeal muscle group, which significantly changed the hydromechanical characteristics of the airway. The adaptation of the upper respiratory tract function and morphology was the fundamental cause of OSAHS. Therefore, when treating OSAHS patients, doctors need to consider how to solve disturbance of pharyngeal muscle group and alleviate the pressure and resistance of airflow in the upper respiratory tract of OSAHS patients, rather than just morphological changes.

The physical model of the upper respiratory tract flow field in this study was obtained from the experimental goats. Experimental studies need to be undertaken at the same time to determine the mechanical features of soft tissue in upper respiratory tract of our people. Based on it, the multi-field coupling analysis, more perfect fluid solid coupling numerical model and multi-scale multi-angle analysis can be performed in succession. Micro-structure discussion can be reached on the basis of macro examination to further understand the pathogenesis of OSAHS.

\section{Abbreviations}


Computational fluid dynamics(CFD)

Obstructive sleep apnea hypopnea syndrome(OSAHS)

\section{Declarations}

\section{Ethics approval and consent to participate}

The picture of ethics approval and consent to participate has been uploaded as the attachment. The animal involved in this study was owned by Jing'an District Zhabei Central Hospital,Shanghai. This study has been approved by insititutional review board (CWO) of Jing'an District Zhabei Central Hospital,Shanghai.

\section{Consent for publication}

The picture of consent for publication has been uploaded as the attachment.

\section{Availability of data and materials}

All data generated or analysed during this study are included in this published article.

\section{Competing interests}

The authors declare that they have no competing interests.

\section{Funding}

The research was all funded by the Shanghai health bureau. The funding body play the main role in collection, analysis, and interpretation of data.

\section{Authors' contributions}

HXY built Computational fluid dynamics(CFD) model on the base of CT scans by 3Dslicer 4.5 and MeshLab and ANSYS ICEM CFD 14.0. SLR, BJ and CJ simulated the internal flow of upper respiratory tract by ANSYS-FLUENT 14.0. ZT, CHH and WLZ analyzed the results by ANSYS-CFD-POST 14.0. HXY was a major contributor in writing the manuscript.All authors read and approved the final manuscript.

\section{Acknowledgements}

Not applicable.

\section{References}

1. White DP, Malhotra A. Obstructive sleep apnoea[J]. The Lancet, 2002,360(9328):237 245. $\otimes$ 
2. Ye Pengcheng, Fang Yiming, Zhang Linlin, et al. Preliminary Study on Finite Element Modeling of Maxillofacial Anatomy Including Chewing System [J]. Journal of Medical

Research,2016,45(4):36 41.

3. Vos W, Partoens B, De-Backer W, et al. Correlation between severity of sleep apnea and upper airway morphology based on advanced anatomical and functional imaging. Journal of Biomechanics[J]. Journal of Biomechanics,2007,40(10):2207

4. Yao Zheng, Chen Kangmin. Overview of CFD General Software [J]. Journal of Shanghai University of Science and Technology,2002,24(2):137 144.

5. Wang Jie, Lin Zhonghui, Li Ying, et al. Research Progress in Computational Fluid Dynamics of Obstructive Sleep Apnea Hypopnea Syndrome [J]. International Journal of Otolaryngology, Head and Neck Surgery,2014,38(5):266 268.

6. Loiseau Jean-Christophe, Naraigh Lennon O, Bethune lain, et al. High-performance computational fluid dynamics: a custom-code approach[J]. A journal of the European Physical Society,2016,37(4):122》

7. Schwab RJ. Upper airway imaging[J]. Clin Chest Med,1998,19(1):33 54.区

8. Kelly JT, Prasad AK, Wexler AS. Detailed flow patterns in the nasal cavity[J]. J Appl Physiol (1985), 2000, 89(1): 323 337.

9. Hahn I, Scherer PW, Mozell MM. Velocity profiles measured for airflow through a large-scale model of the human nasal cavity[J]. J Appl Physiol (1985),1993,75(5):2273 2287.

10. Sun Xiuzhen, Yu Chi, Liu Yingxi, et al. Three-dimensional Finite Element Reconstruction of Human Upper Respiratory Tract and Numerical Simulation of Flow Field [J]. Aerospace Medicine and Medical Engineering,2006,19(2):129 133.

11. Pae EK, Yu YS, Hwang CJ, et al. Customized three-dimensional computational fluid dynamics simulation of the upper airway of obstructive sleep apnea[J]. Angle Orthodontist,2006,76(5):791 799.

12. Wang S, Tu J, Inthavong K, et al. Numerical simulations for detailed airflow dynamics in a human nasal cavity[J]. Respir Physiol Neurobiol,2008,161(2):125 135.

13. Georgalas, C. The role of the nose in snoring and obstructive sleep apnoea: an update [J]. Eur Arch Otorhinolaryngol,2011,268(9):1365 1373.

14. Loth $S$, Petruson $B$, Wiren $L$, et al. Better quality of life when nasal breathing of noringmen is improved at night[J]. Arch Otolaryngol Head Neck Surg,1999,125(1): 64 67.

15. Skitarelic NP, Skitarelic NB, Mladina R, et al. The human external nose and its evolutionary role in the prevention of obstructive sleep apnea[J]. Otolaryngol Head Neck Surg,2010,143(5):712 713.

\section{Figures}



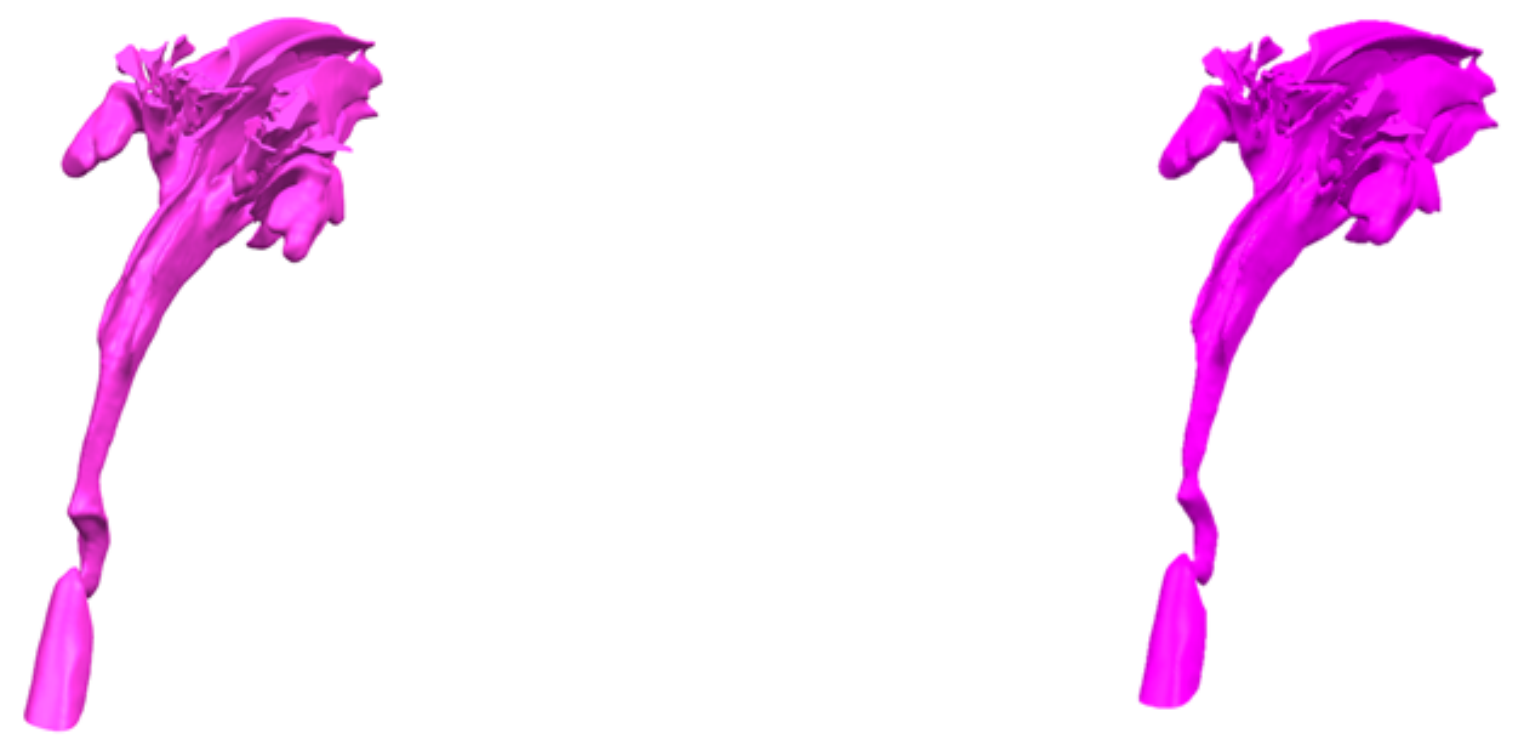

A: Before injection of hardener and submucosal injection edema B: 1 week after injection

\section{Figure 1}

Three-dimensional reconstruction model of respiratory cavity in goats' high simulation of the wall flow field

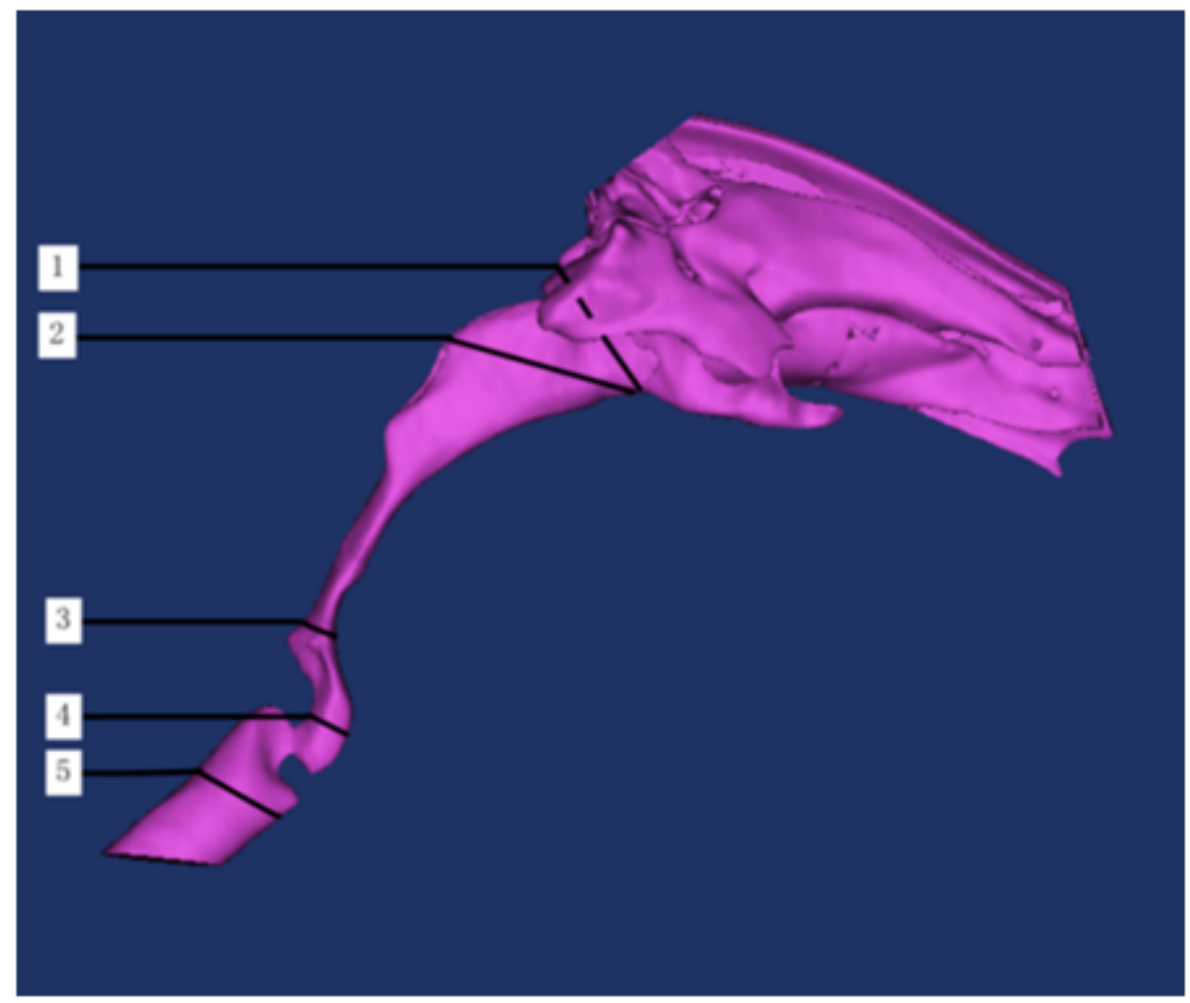

Figure 2 
The selected section of goat CFD model

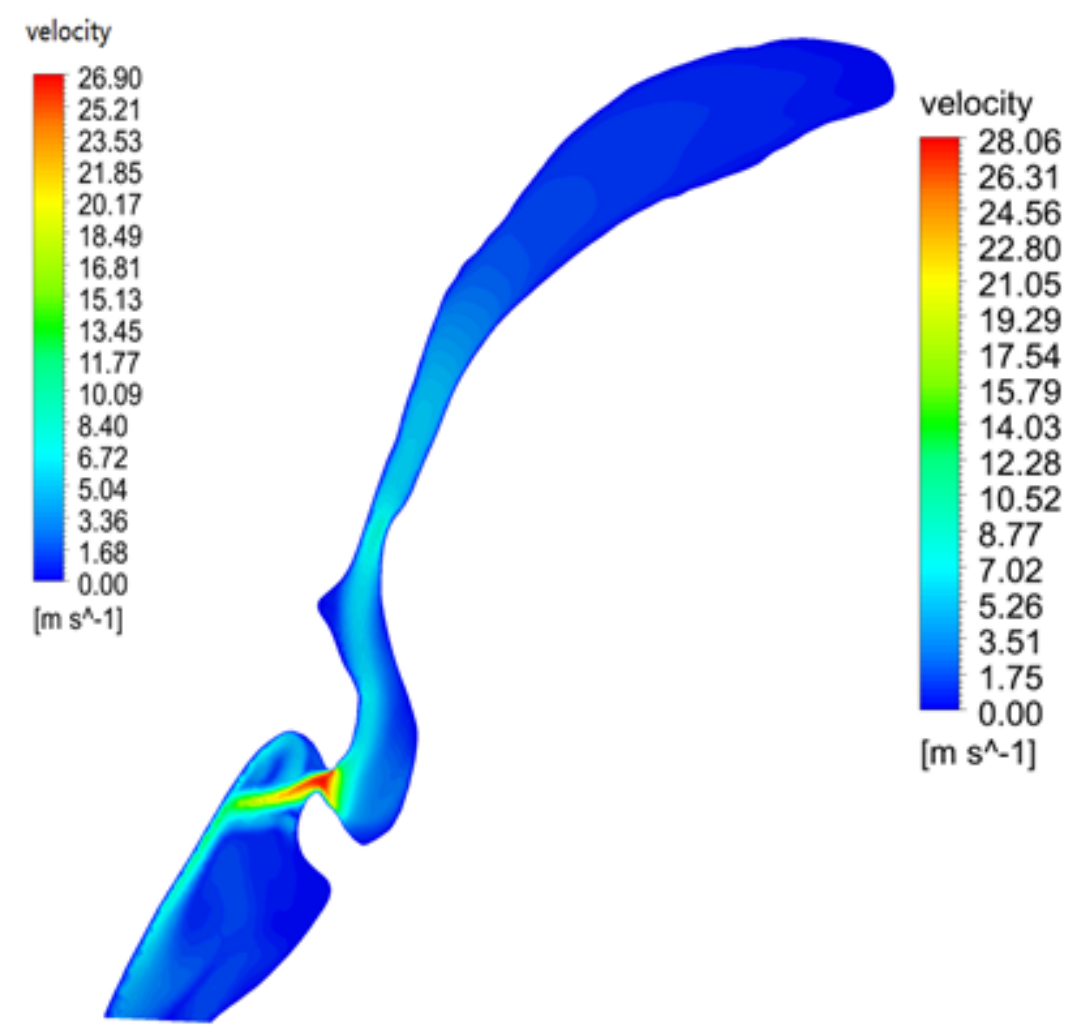

A: Before

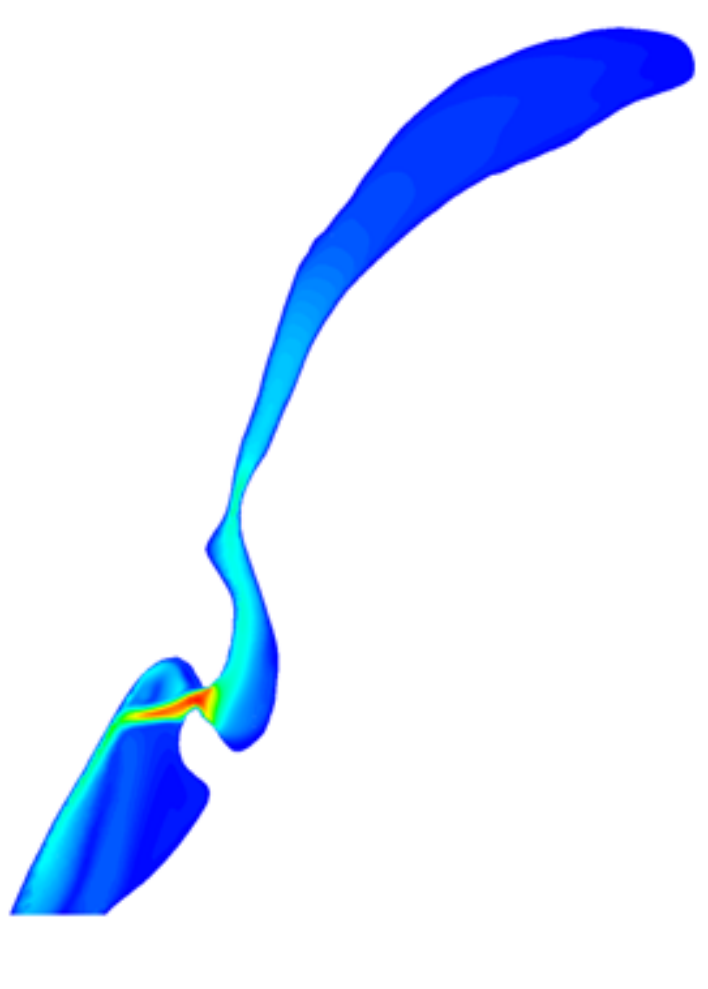

B: After

\section{Figure 3}

Vector diagram of airflow velocity in the midsagittal plane of upper respiratory tract 


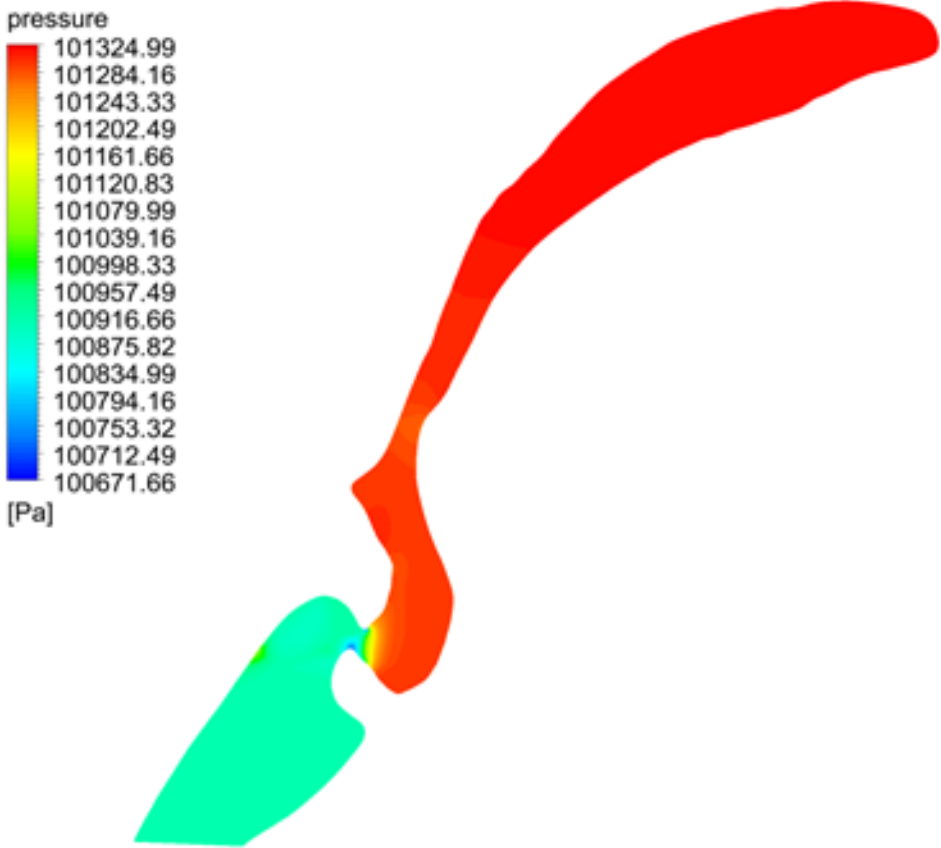

A: Before
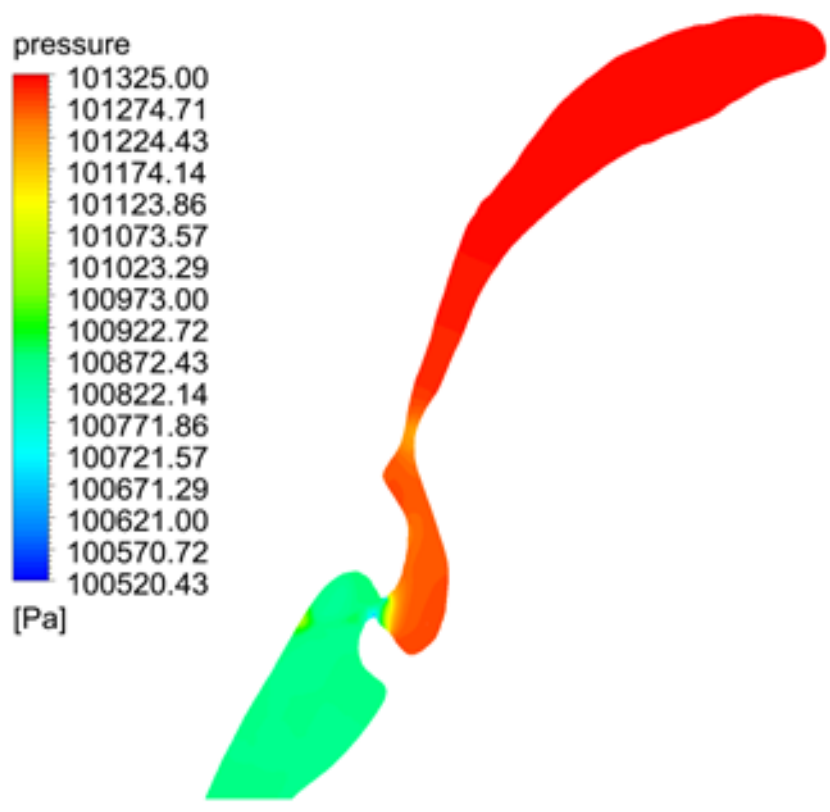

B: After

\section{Figure 4}

Sagittal plane pressure cloud diagram of goat upper respiratory tract 